CrossMark \& click for updates

Cite this: J. Mater. Chem. B, 2015, 3, 4979

Received 26th February 2015 Accepted 30th April 2015

DOI: $10.1039 / c 5 t b 00382 b$

www.rsc.org/MaterialsB

\section{Organic bioelectronics in infection}

\author{
Susanne Löffler, Ben Libberton and Agneta Richter-Dahlfors*
}

\begin{abstract}
Organic bioelectronics is a rapidly growing field of both academic and industrial interest. Specific attributes make this class of materials particularly interesting for biomedical and medical applications, and a whole new class of biologically compatible devices is being created owing to structural and functional similarities to biological systems. In parallel, modern advances in biomedical research call for dynamically controllable systems. In infection biology, a progressing bacterial infection can be studied dynamically, at much higher resolution and on a smaller spatial scale than ever before, and it is now understood that minute changes in the tissue microenvironment play pivotal roles in the outcome of infections. This review merges the fields of infection biology and organic bioelectronics, describing the ability of conducting polymer devices to sense, modify, and interact with the infected tissue microenvironment. Though the primary focus is from the perspective of bacterial infections, general examples from cell biology and regenerative medicine are included where relevant. Spatially and temporally controlled biomimetic in vitro systems will greatly aid our molecular understanding of the infection process, thereby providing exciting opportunities for organic bioelectronics in future diagnosis and treatment of infectious diseases.
\end{abstract}

\section{Introduction}

The healthy organism represents a complex and heterogeneous environment in which a state of equilibrium is maintained. Abrupt changes in the cellular microenvironment are often

Swedish Medical Nanoscience Center, Department of Neuroscience, Karolinska Institutet, SE-171 77 Stockholm, Sweden. E-mail: Agneta.Richter.Dahlfors@ki.se; Fax: +4683426 51; Tel: +46852487425 related to disease. Particularly during infection, the host mounts remarkably complex, concerted responses in order to contain microorganisms to the infection site and to clear the infection. ${ }^{1-3}$ While traditional infection research often applies reductionist approaches, focussing on one or a few variables at a time, the emerging research field tissue microbiology aims to encompass all aspects of the multivariate interactions occurring between the microbe and the host in the complex in vivo environment. ${ }^{4-6}$ Data from live in vivo models have become a valuable source of insight,

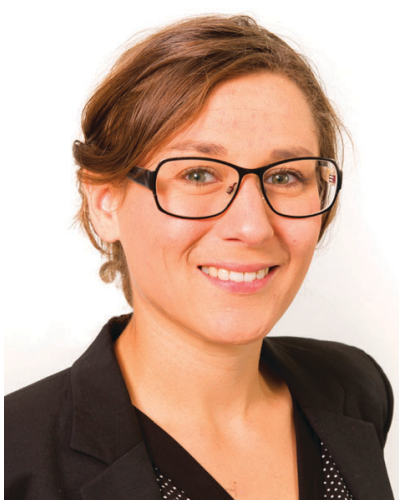

Susanne Löffler
Dr Susanne Löffler has an interdisciplinary background in neuroengineering (biosignal processing) and neurochemistry, promoting the application of engineering sciences in biomedical applications. She earned her PhD in 2012 from the Department of Computer Sciences/Engineering at the University of Lübeck, and worked as a research assistant in experimental neurochemistry at the Department of Neurology, the University Hospital Schleswig-

Holstein. Funded by a fellowship from the German Academic Exchange Service, she moved to the Swedish Medical Nanoscience Center at Karolinska Institutet in Stockholm, where she now is enrolled as a postdoctoral fellow.

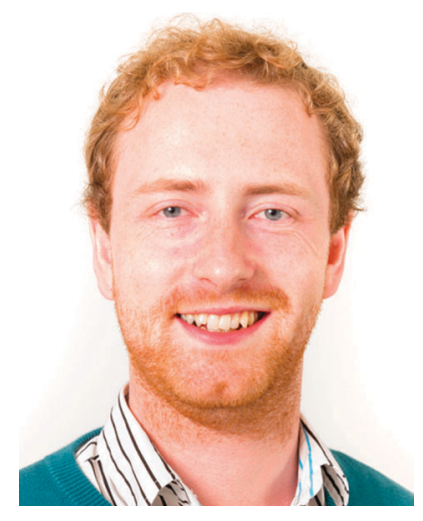

Ben Libberton
Dr Ben Libberton has a background in microbial ecology. He obtained his $\mathrm{PhD}$ from the University of Liverpool where he studied the interactions between bacteria that commonly live on humans. After graduation he began to investigate how the study of infectious bacteria interfaced with other fields, particularly electrical engineering and chemistry. Now at The Swedish Medical Nanoscience Center at Karolinska Institutet in Stockholm, he aims to understand how Organic Bioelectronics can be used to treat and monitor infection, as well as teach us more about the infection process. 
however, maximum extraction of information associated with the progression of infection is hampered due to shortage of useful tools.

Conjugated polymers represents a class of material which, over the last decade, has gained substantial interest for use in biomedical research and medical applications. ${ }^{7-10}$ From a biological and chemical perspective, it seems intuitive to employ carbon-based polymers in medical applications due to their kinship with proteins, carbohydrates and nucleic acids, the building blocks of organic life. Functionalization and adaptation of conjugated polymers using the organic chemistry toolkit further enhance the wide applicability of this material when modelling the properties of cells and tissues. Due to structural and functional similarities to biological systems, organic bioelectronics are used to improve current bio-interfaces and show potential for future applications in the medical field. ${ }^{11}$

This review will highlight recent advances in organic bioelectronics, focussing on applications to (i) multicellular models, (ii) simulation of specialized microenvironments, (iii) monitoring epithelial barrier integrity, (iv) mechanotransduction, and (v) treatment of infections. Primarily, biological applications will be discussed from the perspective of bacterial infections, while also including general examples from cell biology where relevant.

\section{Structural and functional material properties relevant in biological systems}

Conducting polymers exhibit numerous features making them highly compatible with standard methods in modern biological research. The general description of such features and their importance for bio-interactions given in this section is presented in Fig. 1, illustrating the fundamentals of the material,

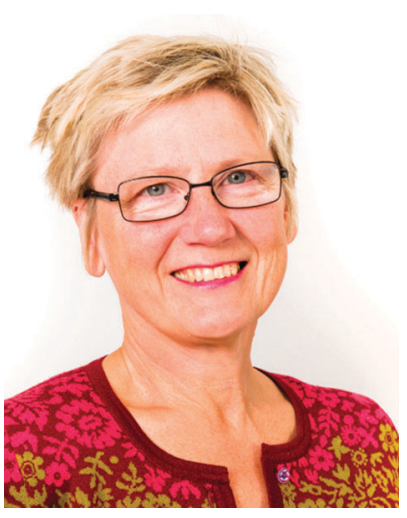

Agneta Richter-Dahlfors
Professor Agneta Richter-Dahlfors is the director of the Swedish Medical Nanoscience Center at Karolinska Institutet, Stockholm. Her pioneering work on real-time intravital imaging of bacterial infections inside the organ formed the basis of the novel area "tissue microbiology". Inspired by the complex environment and physiological challenges bacteria meet inside the host, her interdisciplinary research group generates novel in vitro and in vivo techniques that take the complex pathophysiology of infection into account. She has been active in the field of organic bioelectronics for more than a decade, and uses her complementary expertise in medicine and conductive polymers to develop new communication interfaces between organic electronics and cell/tissue. such as the chemical structure and the basis of their electronic conductivity. This interdisciplinary review provides an overview of the field of organic bioelectronics combined with infection research. Readers with a deeper interest in theory of electrical conductivity or the variety of conducting polymers are referred to more detailed literature. ${ }^{12-16}$

\subsection{Flexibility, transparency}

The structural flexibility of conducting polymer materials allows seamless integration into existing experimental setups commonly used in biomedical research. Their transparency and tuneable optical properties make most conductive polymers conducive to various forms of microscopy, which represent well-established techniques for analysis of a vast number of cellular events. The ability to perform light microscopy and fluorescence-based microscopy on cells cultivated on top of the conducting polymer surface is therefore highly advantageous.

\subsection{Semiconductivity}

In their un-doped ground state, conjugated polymers have semiconducting properties, facilitated by small band gap energies in the range of the visible spectrum. The semiconducting properties are often associated with specific optical properties, which can be modulated by chemical tuning of the molecule in order to adjust the band gap energy. Generally, more planar polymer structures feature smaller band gap energies, which generally fall within the energy spectrum of visible light ${ }^{17-19}$ (see panels (a) and (b) in Fig. 1).

\subsection{Electrical conductivity}

By introducing charge carriers along the polymer backbone, electrical conductivity of semiconducting conjugated polymers can be enhanced. In analogy to common semiconducting materials, conductivity can be increased by several orders of magnitude through a process called doping. ${ }^{15}$ The most common method is p-doping, accomplished by the oxidation of the polymer backbone and subsequent association of negatively charged counter ions. The p-doped conducting polymer can thus be regarded as an ionic complex formed by the oxidized polymer and its counter-anion ${ }^{20}$ (see panels (c) and (d) in Fig. 1).

\subsection{Redox properties}

The redox properties of conducting polymers provide unique features for this class of materials. Low redox potentials of conducting polymer backbones in aqueous electrolyte allow reversible oxidation and reduction of the material in response to an electronic trigger. Switching between redox states of conducting polymers results in reversible modulation of surface energy, topography, wettability, surface charge and stiffness. ${ }^{16,21,22}$ Thus, conductive polymers can be used as electronically controlled active surface switches, which guide cell behaviour in biomedical applications. ${ }^{23-25}$

\subsection{Ionic conductivity}

The translation between electrical currents and ionic flow is a unique property of conducting polymers. The ionic conductivity is based on the structure of conducting polymers being an 

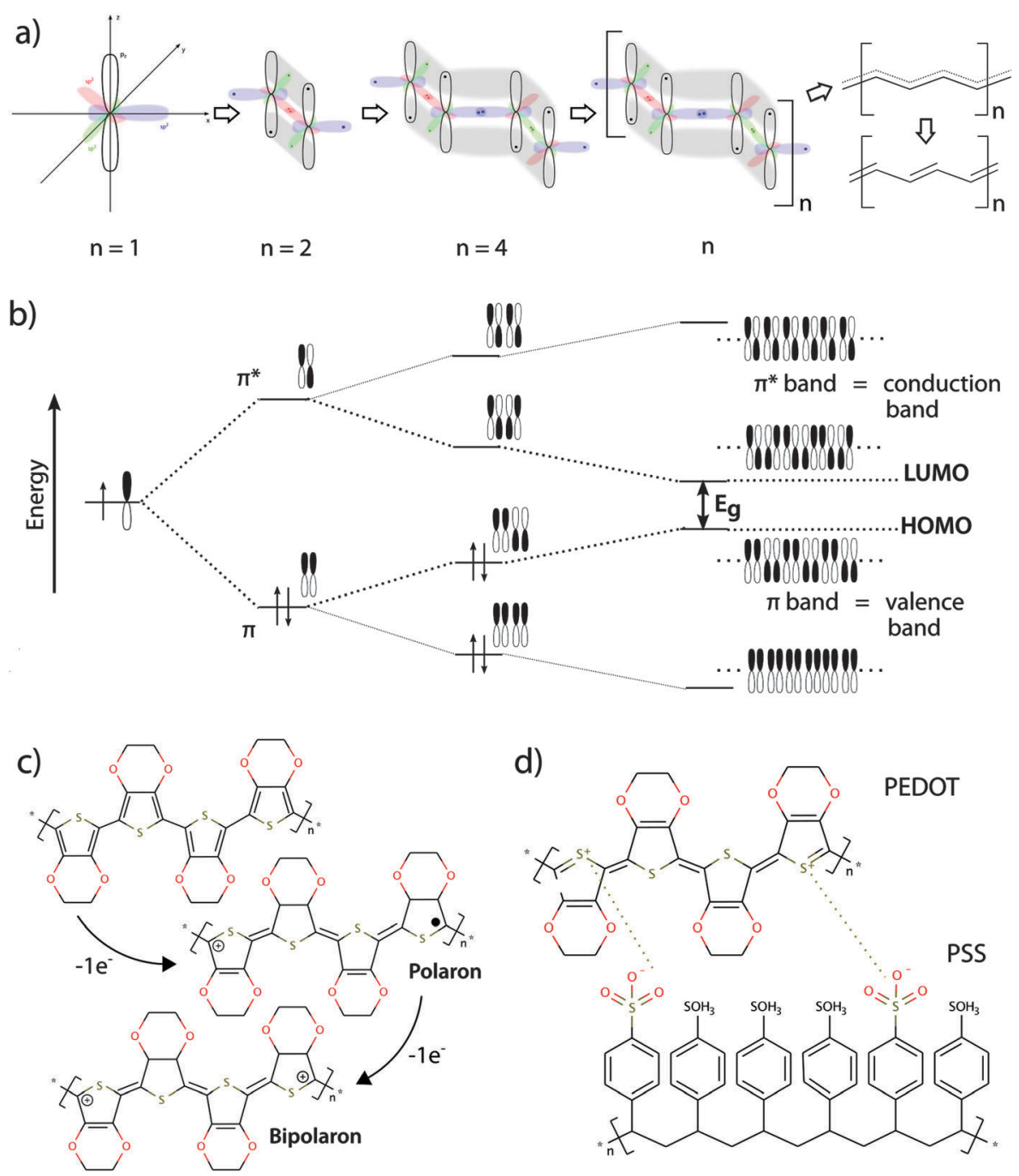

Fig. 1 (a) Conjugated polymers form the basis of organic electronics. A structural hallmark of this class of polymers is their sp ${ }^{2}$-hybridized backbones. Hybridization of perpendicularly orientated $p_{z}$ orbitals allows for binding of electrons not strictly located between two nuclei. Rather, they exist as a system of delocalized $\pi$-electrons in a polymeric molecule with overlapping $p_{z}$ orbitals. The delocalized state represents, however, an unfavourable state and the $\pi$-electron system mesomerizes into the more stable state of alternating single and double bonds (Peierls theorem). (b) When $p_{z}$ orbitals overlap, linear combination of the wave function leads to two different solutions, which represent bonding $\pi$-orbitals at a lower energy level and anti-bonding $\pi^{\star}$-orbitals at a higher energy level. As the polymer chain becomes longer ( $n$ increases), hybridization occurs and the more $\pi$ and $\pi^{\star}$ energy levels are created. Eventually, the energy differences between the different levels become so small that energy bands are formed within the $\pi$ - or $\pi^{\star}$-levels. The available energy levels are filled with electrons according to the Pauli principle, with the lower energy levels being filled first. Therefore, the $\pi$-band is fully occupied while the $\pi^{\star}$-band is empty. The lowest unoccupied energetic level is called "lowest unoccupied molecule orbital" (LUMO) and the highest energetic level containing electrons is called "highest occupied molecule orbital" (HOMO). In analogy to the band model for electrical conductors, semiconductors and insulators, the $\pi$-band represents the valance band and the $\pi^{*}$-band represents the conduction band. A band gap energy $E_{\mathrm{g}}$, which is the energy difference between the HOMO and LUMO level in a conjugated polymer, is necessary for electron transition from the valence band into the conduction band. (c) Full occupancy of the valence band impairs electron movement. The electrical conductivity of conjugated polymers thus needs to be increased by orders of magnitude to be of use in organic bioelectronics. Upon oxidation, removal of one electron from the polymer chain produces a mobile radical cation (polaron). Further oxidization can lead to formation of a spinless bipolaron with immobile positive charges (electron holes), or introduce another polaron. The bipolarons created during oxidation of the polymer ( $p$-doping) can be viewed as electron holes, which increase electron mobility and make the polymer electrically conductive. The example shows p-doping of the conjugated polymer PEDOT. (d) To achieve charge equilibration in the doped polymer, counter-ions are incorporated along the backbone, which are often called dopants. The example shows the doped conductive polymer PEDOT:PSS.

ionic complex. Ion movement into or out of the polymer for the purpose of charge equilibration leads to ionic currents, which are often summarized under the term ionic conductivity. The redox switch of doped conducting polymers leads to electrochemical oxidization or reduction and thus alteration of charges at the polymer backbone. Reduction of a p-doped conductive polymer leads to dissociation of the polymer backbone with its counter-anion (de-doping). Whereas monomeric counter-anions are likely to diffuse out from the polymer, polymeric counterions will be retained in the polymer matrix, which hinders their movement. This effect is illustrated in the example of the conducting polymer poly(3,4-ethylenedioxythiophene) (PEDOT) 
a)

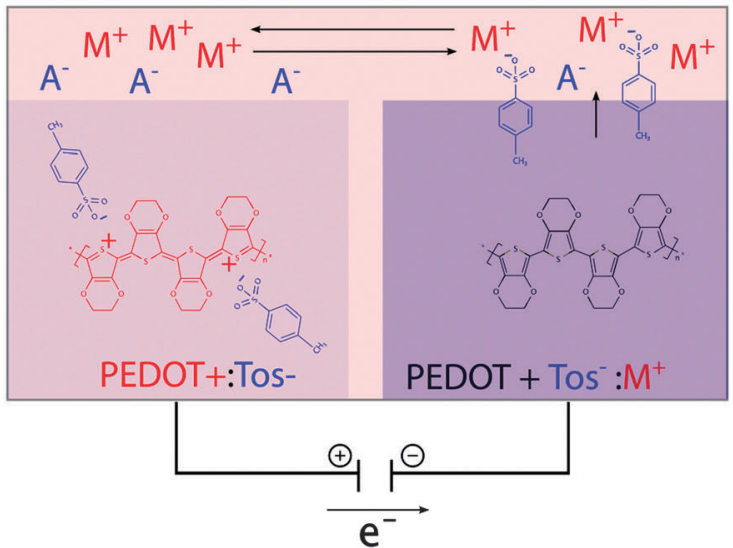

b)

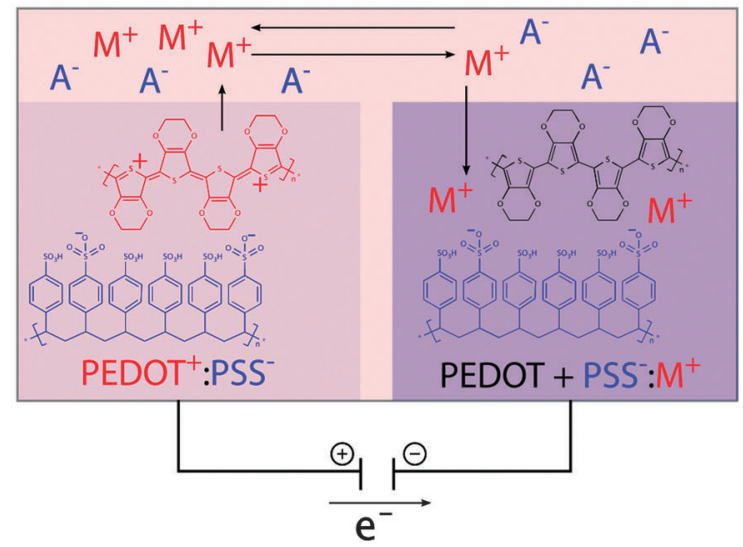

Fig. 2 Ion exchange between oxidized/reduced conducting polymer and electrolyte. (a) The monomeric counter ion Tos ${ }^{-}$diffuses out of the polymer, thereby generating charge equilibration. (b) Movement of the polymeric counter ion PSS ${ }^{-}$out of the polymer is hindered due to its size. Charge equilibration is mainly achieved by cations moving into the polymer.

with the monomeric dopant tosylate (Tos) (see Fig. 2a) and the polymeric dopant polystyrenesulfonate (PSS) (see Fig. 2b). In both cases, charge equilibration is achieved by ions flowing into, or out of the polymer, giving rise to the ionic conductivity. The de-doping process has marked conducting polymers as responsive systems for specialized drug delivery applications. ${ }^{26,27}$ Also, ionic conductivity is highly advantageous when developing biomimetic devices, since it enables precise modulation of local ion homeostasis as a mimic of pathogenesis-associated alteration of the ionic microenvironment.

\subsection{Swelling}

Some conductive polymers show mechanical properties related to polymer swelling. This is due to inflow of hydrated ions for the purpose of charge equilibration after redox switching. Depending on the polymer structure and dopant, swelling can lead to significant dimensional changes with high stress generation and high work capacity per cycle. Polypyrrole (PPy) doped with various compounds has been used to develop electrochemically controlled microactuators. ${ }^{28-30}$

\subsection{Roughness and surface area}

Due to their polymeric structure, conductive polymers possess a large active surface area compared to metal electrodes of similar size. Simple fabrication methods like electro-deposition or vapour phase deposition make it possible to coat porous 3D structures, thereby increasing the active surface area even further. ${ }^{23}$ A significantly larger electrode area can potentially yield better signal-to-noise ratios, enhanced sensitivity, and lower detection limits for various sensing applications. In biosensing, for example, an increased surface area can lead to a greater amount of immobilized reagent on the surface and thus to larger sensor currents. ${ }^{31}$ For a review on various sensing applications of organic bioelectronics, we refer to more comprehensive literature. ${ }^{32,33}$
Collectively, the unique properties of conducting polymers, as compared to traditional materials, offer improved compatibility with cells and tissues. Due to their combined ionic and electronic conductivity, conducting polymers translate between common electronic and biological systems. Thus, organic bioelectronics will likely play an important role in the development of biomimetic in vitro systems to help unravel the complex situation in the infected host organism.

\section{Modelling the complexity of in vivo environments}

\subsection{Multicellular 2D models}

The complex environment of the live host allows bacteria with broad tissue tropism to infect many cell and tissue types. Bacterial colonization is often initiated at a localized site on a mucosal surface. Colonization may occur in the gastrointestinal tract, the upper (nose, throat) and lower (lung) respiratory tract and the urogenital tract (bladder, kidney). Immediate changes in the local microenvironment, induced by the bacterial metabolism or early host immune responses, serves to orchestrate the host response with a goal to clear the infection. Such responses include local production of pro-inflammatory signalling molecules, so called cytokines and chemokines, originating from resident infected and non-infected cells, as well as from cells recruited to the site of infection. ${ }^{34}$ This complex scenario provides a great challenge when constructing in vitro models, aiming to decipher the involvement of different cell types in tissue homeostasis during infection. ${ }^{5,35}$ This has prompted a great interest in the development of improved biomimetic devices and in vitro models. By enabling temporally controlled studies of organized multicellular structures with spatially controlled cell distribution and preservation of cell function, systems are now being developed which will help to solve the puzzle. ${ }^{36}$ 
Novel opportunities offered by early advances in nanotechnology demonstrated how physical surface properties, such as nanotopography, could be used to influence cell behaviour. Cultivation of uroepithelial cells on differently nanostructured surfaces demonstrated altered epithelial cell morphology, associated with differential expression of cytokines and chemokines. ${ }^{37}$ Guidance of morphological adaptation, leading to improved elongation and alignment of smooth muscle cells was reported, ${ }^{38}$ and it was demonstrated how the spacing of patterned RGD (arginine-glycine-aspartate) motifs, the ligands for cell attachment via integrins, affected adhesion, proliferation, and differentiation of mesenchymals stem cells. ${ }^{39}$

Today, the fast-growing research field of organic bioelectronics is providing novel opportunities to create active surface patterns. Electro-active polymer systems offer a major advantage since applying an electrical potential can reversibly modulate the surface properties of these polymers. In patients with pyelonephritis (kidney infection), colonization by uropathogenic $E$. coli on the mucosa of the kidney's tubular system causes major disruption of the tight monolayer of renal epithelial cells. As a consequence, important physiological functions such as reabsorption of ions from the primary urine back into the vasculature are damaged. As a first step to mimic this situation in vitro, patterned organic bioelectronics surfaces were used to generate active control of epithelium formation in a functionalized cell culture dish. ${ }^{25}$ Concurrent with the seeding of renal epithelial Madin-Darby canine kidney (MDCK) cells, an electrical potential was applied to induce changes in the redox state of the conducting polymer system PEDOT:Tos (see Fig. 3a). Within the same cell culture dish, MDCK cells were thriving when attached to the reduced surface, showing normal cell morphology, proliferation, and formation of tight junctions, whereas controlled cell death, so called apoptosis, was observed in cells initially adhering to the oxidised surface. The molecular mechanism governing the difference in cell behaviour is attributed to redox-dependent effects on surface-bound fibronectin (Fn). While conformation of Fn is maintained on the reduced surface, the oxidised surface introduces a conformational change, thereby hiding the RGD motifs from access by the cellular integrin attachment complexes. ${ }^{40}$ Interestingly, continuous redox switching does not influence the functionality of the cells attached to the reduced polymer. This is because the material remains oxidized/reduced as the current flow ceases a few minutes after switching. ${ }^{23,25}$

Other cells, such as fibroblasts, also show tuneable attachment behaviour dependent on the redox state. When grown on a microwrinkled conductive polymer interface, wrinkles in the surface topology were shown to guide anisotropic multicellular alignment of murine skeletal muscle cells on a fibroblasts feeder layer. Additionally, fibroblast attachment and formation of a topographically aligned multicellular layer was prevented on oxidized PEDOT:PSS surfaces. ${ }^{41}$

Due to a variety of cell adhesion mechanisms and expression of extracellular matrix components, it is likely that distinct cell types are influenced in different ways by the redox state of a conductive polymer. As a tool to investigate cells' preferred redox states, an organic electrochemical transistor (OECT) was developed. The OECT, which is a variant of the organic thin film transistor, ${ }^{42-44}$ features a channel exposed to the cells and media while serving to connect the source and drain electrodes. ${ }^{24,45}$ By addressing the OECT, an electrical potential gradient establishes over the channel, which can be tuned by adjusting the gate potential. MDCK cells seeded on the channel were shown to respond to the electrochemical gradient by attachment at preferred positions along the channel (see Fig. 3b). A cell gradient was thus generated with the lowest numbers of adherent cells present on the oxidized end. Cell numbers were steadily increasing towards a)

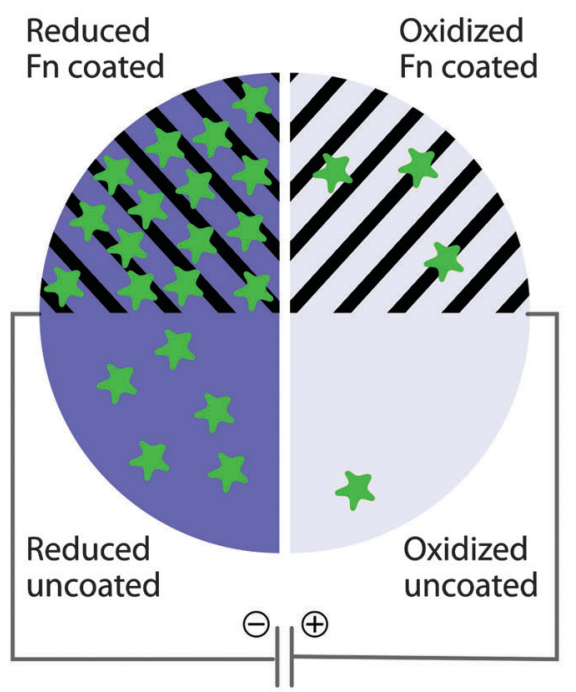

b)

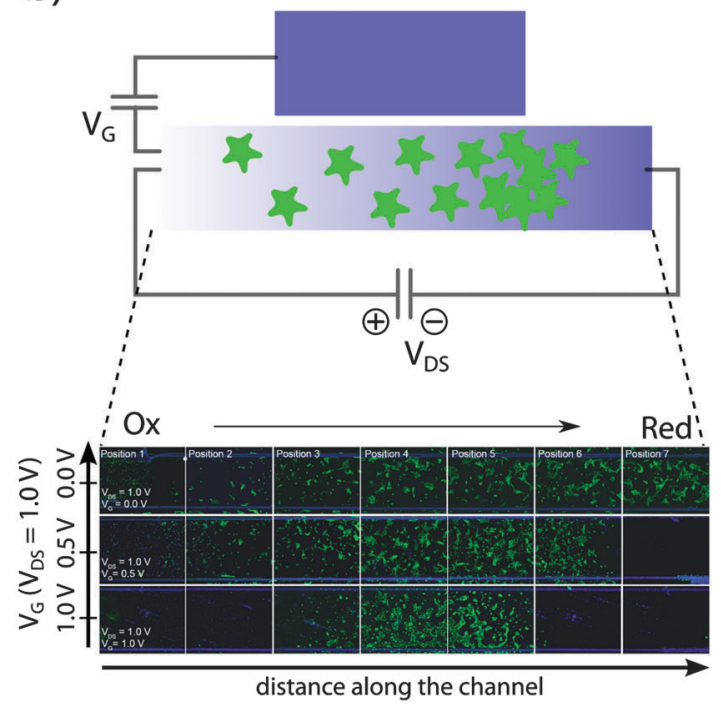

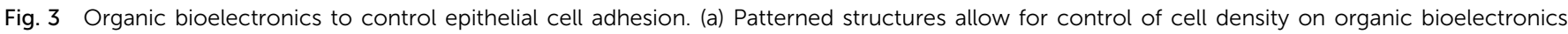

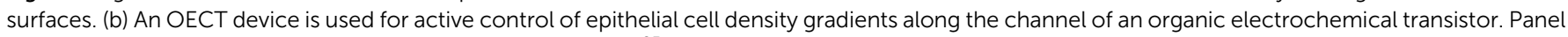
(b) is modified and partly reproduced from Svennersten et al. ${ }^{25}$ 
the reduced end, until an abrupt drop was observed at the highest reduction potential. Increasing the steepness of the gradient by tuning the gate potential creates a narrow band of material redox states, to which cells are able to adhere and proliferate.

Whereas the transistor design of Bolin et al. ${ }^{24}$ favored an exponential gradient of the redox potential, reflected in the cell density pattern, linear redox gradients can also be produced. By coating PEDOT:Tos on top of an indium tin oxide (ITO) film, the redox state of the polymer can be aligned to the linear potential gradient of the underlying ITO. This linear gradient device was developed to produce linear gradients of mesenchymal fibroblasts and fibroblast cell lines. ${ }^{46}$

As a natural extension of on-going work, mapping the preferred surface redox states for different cell types is the next challenge on our way towards elaborate multicellular in vitro models based on electrochemical adhesion matrices made from conducting polymers. We foresee that redox modulation could provide an elaborate tool to produce $2 \mathrm{D}$ patterns to be applied in multicellular in vitro models. Sequential electrochemical surface switching combined with timely seeding of different cell types would allow for correct positioning of different, functionally related cell types in the same cell culture dish. These systems would serve as excellent tools to decipher intercellular signalling pathways, as exemplified by epithelial cells of infected mucosa communicating with the endothelial cells of the vasculature.

\subsection{Towards multicellular 3D models}

An emerging body of evidence shows that the 3D organisation of tissues, organs and even organ systems play a vital role in infection. ${ }^{34,47}$ Extending the concept of horizontal multicellular $2 \mathrm{D}$ cell culture models to $3 \mathrm{D}$ tissue models requires techniques to release a cell layer from the surface on which it has grown. Stacking of cell layers with defined multicellular 2D structure have the potential to evolve to fully developed 3D tissue or organ models in which intercellular communication and the tissue response to infection can be studied under realistic conditions.

Electro-responsive systems for cell sheet technology have been developed, based on the affinity of cells to the RGD peptide as attachment anchor. In one example, the RGD peptide motif is presented to the cells via an electro-active tether to a selfassembled monolayer (SAM) of alkenethiolates on Au. ${ }^{48}$ The electro-active tether contains a quinone ester, which is reduced to hydroquinone upon application of an electrical potential to the underlying Au. Rapid cyclization of hydroquinone releases the RGD peptide to which cells are attached via integrins. A potential of $-0.7 \mathrm{~V}$ applied via the Au substrate was shown to release fibroblasts evenly distributed over the entire electro-active surface. Reversing this principle by addition of a cyclopentadiene modified RGD motif during oxidation of the hydroquinones to quinones results in immobilization of the RGD peptide, altering (switching) a surface from a state that prevents to a state that promotes cell attachment. This mechanism has been used to "switch-on" and synchronize the migration of fibroblasts attached to specific locations on a patterned surface. ${ }^{49}$ As alternative to quinones, thiols can be used as electro-active groups tethering the RGD peptide to a SAM on a Au surface. ${ }^{50}$ Application of this technology on Au threads has been used to engineer capillary-like structures as tissue constructs. ${ }^{51}$

Based on the finding that polyelectrolyte mono- and multilayers desorb upon electrochemical polarization of the substrate ${ }^{52}$ a polyelectrolyte system coated on the conductive polymer ITO has been used to release sheets of osteoblasts and fibroblasts after the application of a positive potential to the ITO layer. ${ }^{53}$ Applying the earlier described polyelectrolyte technology on a micropatterned ITO platform, it was possible to obtain micropatterned co-cultures of primary human chondrocytes and human mesenchymal stem cells. ${ }^{54}$

A novel strategy for cell release was recently presented based on a self-doped water-soluble conducting polymer. ${ }^{55}$ The backbone structure of the newly developed polymer PEDOT-S:H resembles that of PEDOT, one of the most stable conducting polymers that is in use today. ${ }^{56}$ In the self-doping PEDOT-S:H, negatively charged sulfonate groups are linked to the backbone's thiophene group via alkyl chain arms. In a reduced state, the polymer associates with $\mathrm{H}^{+}$to balance its internal negative charge. By applying an electrical potential that oxidizes the thiophene, $\mathrm{H}^{+}$are expelled and the negatively charged groups on the alkyl arms are drawn to the center of the molecule. This results in a major structural change, eventually leading to swelling, cracking and finally disaggregation of the polymer. Key structural properties include a very high degree of intermolecular selfdoping, which gives good adhesive properties to different substrates and high conductivity $\left(30 \mathrm{~S} \mathrm{~cm}^{-1}\right)$. By incorporating PEDOT-S:H thin-film electrodes into customized, optically transparent cell culture devices, human uroepithelial bladder cells were released upon electrochemical trigger. Released cells showed high viability and were functionally intact. Importantly, cell surface antigens were preserved to a greater extent when this method was used as compared to traditional trypsination. The PEDOT-S:H method combines high spatial resolution of detachment, green synthesis and electrochemically triggered release of cells from a surface. Automated, local detachment of cells based on conductive polymer cell culture substrate may serve as important tools when studying epithelial exfoliation and the initiation of wound healing processes. ${ }^{55}$

\subsection{Mimicking specific microenvironments in niches of infection}

Bacterial pathogens are adapted for colonization and proliferation within a variety of tissues. This tropism depends on the specific microenvironment in each location, which changes as the infection progresses. Controlled reconstruction of such dynamic environments in vitro is a difficult task. However, a growing toolbox of organic bioelectronic devices with high level of spatiotemporal control offers novel opportunities to facilitate in vitro studies of infections under more realistic conditions.

The stomach is a special microenvironment, in which gastric epithelial cells are exposed to highly acidic conditions and degrading enzymes, while efficiently protecting themselves from autodigestion. Acidity is caused by proton $\left(\mathrm{H}^{+}\right)$secretion in the gastric glands by $\mathrm{H}^{+} / \mathrm{K}^{+}$-ATPases in the cell membrane. ${ }^{57}$ 
a)

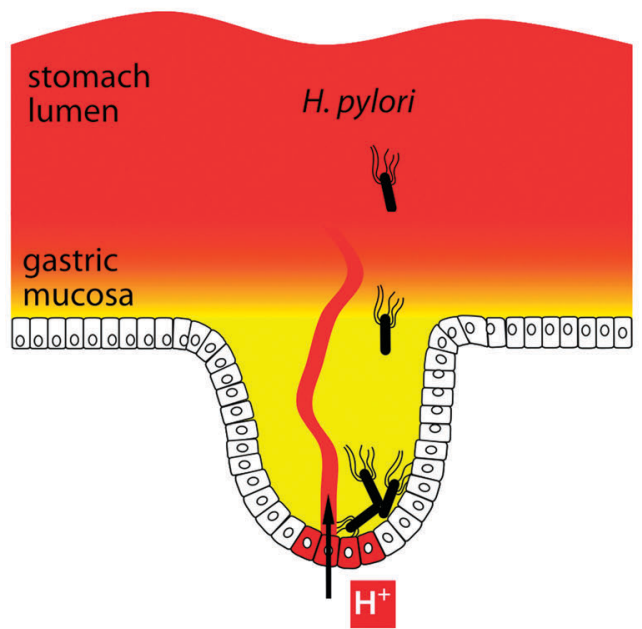

c)

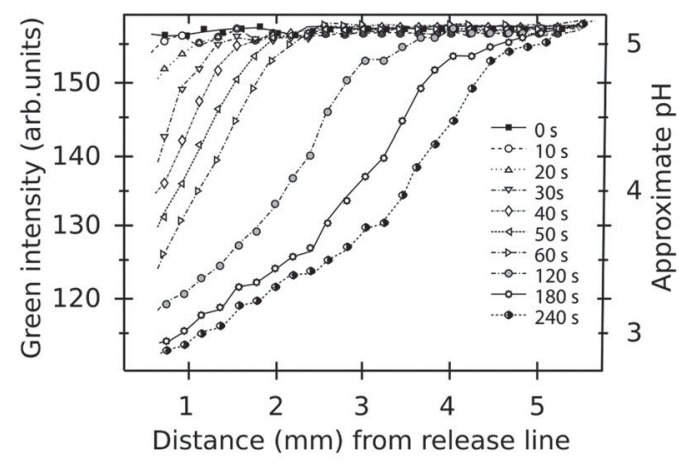

b)

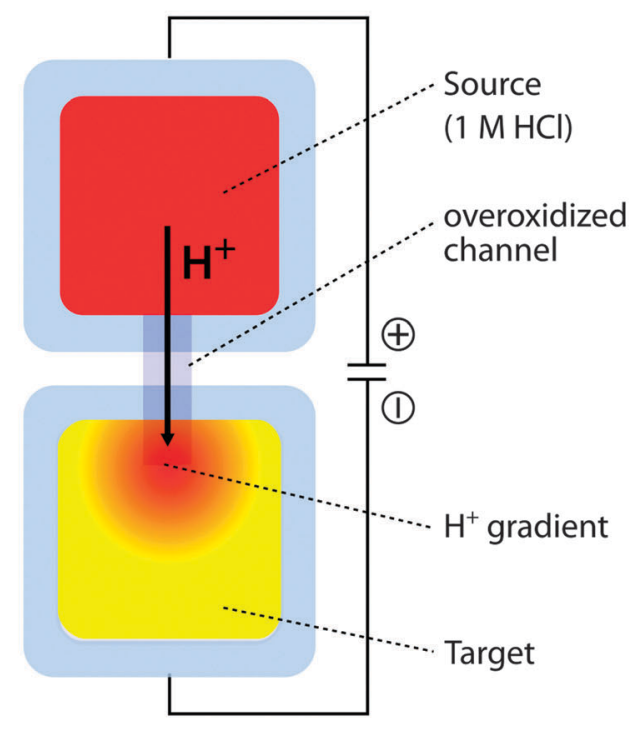

d)

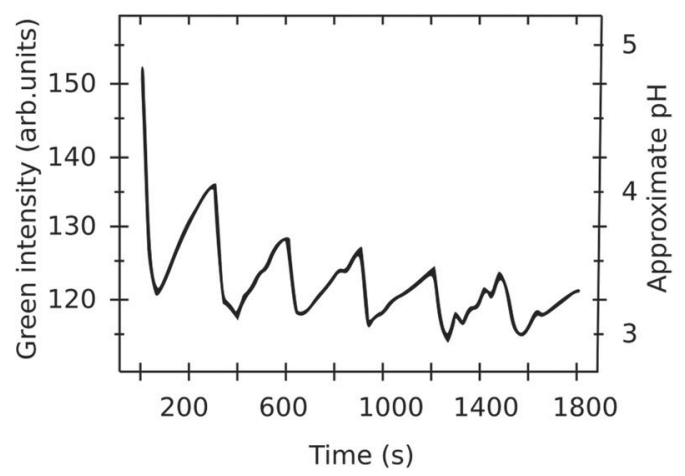

Fig. 4 Simulation of $\mathrm{pH}$ gradients in the gastric mucosa using the OEIP. (a) H. pylori colonizing the gastric epithelium despite a protecting pH gradient (red to yellow) in the gastric mucosa. (b) Generation of a pH gradient (red to yellow) in the target electrolyte by controlled transport of $\mathrm{H}^{+}$through the overoxidized PEDOT:PSS channel in an OEIP. (c) Characterization of the $\mathrm{pH}$ gradient in the OEIP target electrolyte as a function of increased $\mathrm{H}^{+}$transport times. (d) $\mathrm{pH}$ oscillations in the target electrolyte are created by addressing the OEIP with pulses of $15 \mathrm{~s}$ and $10 \mathrm{~V}$. Panels (c) and (d) are modified or reproduced from Isaksson et al. ${ }^{63}$

$\mathrm{H}^{+}$is relayed to the stomach lumen via mucus layer channels. This generates $\mathrm{pH}$ gradients with neutral to slightly alkaline $\mathrm{pH}$ closest to the epithelial surface, and acidic $\mathrm{pH}$ of 2-3 in the luminal part (see Fig. 4a). ${ }^{58,59}$ Despite high efficiency of $\mathrm{pH}$ barriers in defence against infection, some bacteria, such as Helicobacter pylori (H. pylori) have evolved to overcome this challenge. Membrane-bound chemoreceptors sense the external environment, instructing bacteria to move away from the acidic centre towards the peripheral crypts, where proliferation of $H$. pylori has been linked to diseases such as stomach ulcers and cancer. ${ }^{60-62}$

Organic bioelectronic devices have been developed that now allow us to create ionic gradients resembling the acidic gradient in the gastric mucosa. An organic electronic ion pump (OEIP) was developed based on the chemically stable polymerpolyelectrolyte system PEDOT:PSS. ${ }^{63,64}$ As a consequence of its electrochemical properties (see Section 1) the material can conduct both electrons and ions. In the OEIP device, a PEDOT:PSS film is patterned onto a polyethylene terephthalate (PET) substrate (see Fig. 4b). The pattern consists of two electrodes, a source and a target, connected by an overoxidized channel. Due to overoxidization, the channel is electronically insulating, but can conduct ionic currents. Patterning of the hydrophobic photoresist SU-8 creates electrolyte reservoirs over the PEDOT:PSS source (anode) and target (cathode) electrodes. The source electrolyte contains the ions that are to be transported and the target electrolyte contains a counter-electrolyte, which can be chosen according to application e.g. cell culture medium, any kind of buffer or even extracellular fluid for in vivo applications. ${ }^{65}$ When the OEIP is addressed with an electrical potential between source and target electrode, electrochemical reactions occur (see eqn (1)). Oxidation of the PEDOT:PSS on the anode results in ions being electrophoretically transported through the channel towards the cathode target electrolyte. 
Anode (source): PEDOT + PSS $^{-}+\mathrm{M}^{+} \rightarrow$ PEDOT $^{+}:$PSS $^{-}+\mathrm{e}^{-}+\mathrm{M}^{+}$ Cathode (target): PEDOT $^{+}: \mathrm{PSS}^{-}+\mathrm{e}^{-}+\mathrm{M}^{+} \rightarrow$ PEDOT $+\mathrm{PSS}^{-}+\mathrm{M}^{+}$

A unique feature of the OEIP is that $\mathrm{H}^{+}$is delivered without causing convection, thereby minimizing any disturbance in the target electrolyte. $\mathrm{pH}$ gradients stretching across a distance of up to $4 \mathrm{~mm}$ can be easily established and maintained, with a steepness controlled by the applied voltage (see Fig. 4c). ${ }^{66}$ The OEIP device thus serves as an excellent tool to facilitate advanced studies of $H$. pylori invasion into the gastric mucosa and its capability to evade host defence mechanisms.

Generally, ion gradients or oscillations of ion concentrations are important driving forces in biological systems. In kidney infections, regular fluctuations of the intracellular $\left[\mathrm{Ca}^{2+}\right]$ in renal epithelial cells were shown as critical for initiation of the host innate immune response. ${ }^{67-69}$ The signal is initiated by the bacterial toxin $\alpha$-hemolysin (Hly) produced by uropathogenic E. coli colonizing the proximal tubule of the nephron. ${ }^{69}$ Hly triggers a cascade of signalling events in the cell. Activation of voltage-operated $\mathrm{Ca}^{2+}$ channels in the plasma membrane leads to $\mathrm{IP}_{3}$-receptor regulated release of $\mathrm{Ca}^{2+}$ from internal stores. Concerted efforts of cellular systems act in synergy to establish a temporally well-controlled oscillation of the intracellular $\left[\mathrm{Ca}^{2+}\right]$. Being one of the most versatile and universal signalling agents, the $\mathrm{Ca}^{2+}$ ion is known to regulate many different cellular processes, and specificity is attributed to frequency and amplitude of the $\mathrm{Ca}^{2+}$ response. ${ }^{70}$ The effect of Hly on renal cells provides the first example describing the influence of a bacterial toxin on eukaryotic gene transcription by frequency-controlled $\mathrm{Ca}^{2+}$ signalling. ${ }^{67}$ Slow oscillations at a frequency of $1.4 \pm 0.1 \mathrm{mHz}$, corresponding to a periodicity of $12 \pm 0.7 \mathrm{~min}$, was shown to regulate the production of pro-inflammatory chemokines IL-6 and IL-8 in renal epithelial cells. ${ }^{67,71,72}$

Changing the local ionic microenvironment while maintaining the overall ionic strength of the medium is extremely difficult in traditional in vitro systems. Fast diffusion in fluidic environments prevents maintenance of high local concentrations, and generation of amplitude- and frequency-controlled ionic oscillations is unattainable. Ion transport over the OEIP channel is, however, fast compared to the rate of diffusion in the target electrolyte, making high local concentrations at the channel outlet attainable. ${ }^{63,64}$ In the off state, when no potential is applied over the channel, ion transport stops. As a consequence, the local state of high ion concentration is diminished due to diffusion in the target electrolyte. The overall concentration in the medium will, however, remain almost unchanged, since the number of transported ions is negligible compared to the bulk concentration. A series of publications demonstrate the use of the OEIP device in modulating the ionic microenvironment and in establishing ionic oscillations with defined frequency and amplitude. $^{63,64}$ Matching suitable pulse duration schemes (on/off times) with ion diffusion in the electrolyte create ionic oscillations with periodicities highly relevant in biological systems (see Fig. 4d) ${ }^{70,73}$ In addition to mono-and divalent cations, other signalling substances can be delivered given they are positively charged during transport.
Examples include aspartate, glutamate, GABA and acetylcholine (ACh) ${ }^{65,74}$ Miniaturizing the transport channel to $10 \mu \mathrm{m}$ width, spatial control at the single cell level is achieved. In ACh-responsive neuronal cells located $50 \mu \mathrm{m}$ apart, transported ACh elicits selectively a $\mathrm{Ca}^{2+}$ response in one cell but not the other. By varying the duration of the transport pulses from $0.2-2 \mathrm{~s}$, precise control of the amplitude of the cell response, as well as temporal control of the intracellular $\mathrm{Ca}^{2+}$ oscillations is achieved. ${ }^{74}$

An example highlighting the importance of understanding effects of the local ionic microenvironment is activation of the C-reactive protein (CRP). This acute phase mediator of the innate immune system is extensively produced by liver hepatocytes in response to infection, and accumulates in high concentrations in the systemic circulation. Human CRP shows high affinity to phosphocholine (PC) residues, as well as other autologous or extrinsic ligands. ${ }^{75}$ Goda et al. hypothesized that the ionic conditions at an infection site promote local CRP recruitment and activation from a large pool of systemically circulating CRP. ${ }^{76}$ By integrating a PC biomimetic receptor membrane in the OEIP, the ion-dependency of CRP binding to its receptor was analyzed in real-time by total internal reflection fluorescence (TIRF) microscopy. Being a high-resolution imaging system, TIRF microscopy is highly sensitive to any disturbing factors. The ability to transport ions without bulk liquid flow was therefore critical to enable gradual alteration of the $\mathrm{pH}$ and $\left[\mathrm{Ca}^{2+}\right]$ during TIRF imaging. A dynamic activation of CRP on the PC biomimetic receptor membrane was observed which indeed was dictated by a spatiotemporal change in ion homeostasis. ${ }^{76}$

\subsection{Monitoring epithelial barrier integrity}

Specialized epithelial cells constitute barrier surfaces separating mammalian hosts from the external environment. ${ }^{77-79}$ Being the main regulator of tissue homeostasis with the outside environment also comes with adverse effects, making the epithelium vulnerable as an entry point for pathogens. Primary determinants of the epithelial barrier function are plasma membrane integrity, and intercellular tight junction. During infection, bacterial strategies to circumvent the barriers are counteracted by host mechanisms, acting to clear the infection with minimal damage imposed on the healthy tissue and re-establishment of tissue homeostasis (see Fig. 5a).

The dynamic interplay between bacteria and the infected host is at the focus of the recently established area tissue microbiology. ${ }^{4-6}$ Intravital microscopy, often based on non-invasive multiphoton (2-photon) microscopy, has been demonstrated as an excellent tool to study the dynamics of infection within the host. In a pioneering model of intravital infection studies, colonization of uropathogenic $E$. coli on the rat kidney epithelium was visualized in real-time during the first eight hours of infection. ${ }^{69}$ The efficient colonization of normal tissue is accompanied by loss of epithelial polarity and blebbing of epithelial cells. Interestingly, clotting is initiated in the nearby vasculature leading to induction of ischemia and ischemic injury. ${ }^{80}$ Having the capacity to grow under aerobic as well anaerobic conditions, bacteria still multiply despite a hypoxic environment, and ischemia-associated sloughing of epithelial cells enables their paracellular migration. Simultaneously, signals 
a)

Healthy epithelium:

- limited ion exchange

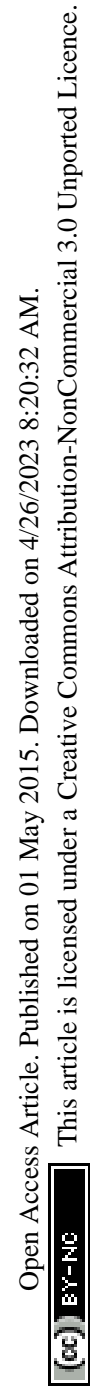

b)

c)

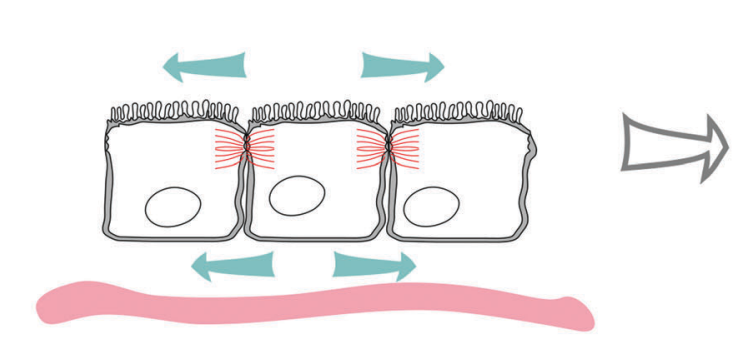

Infected epithelium:

- unhindered paracellular diffusion
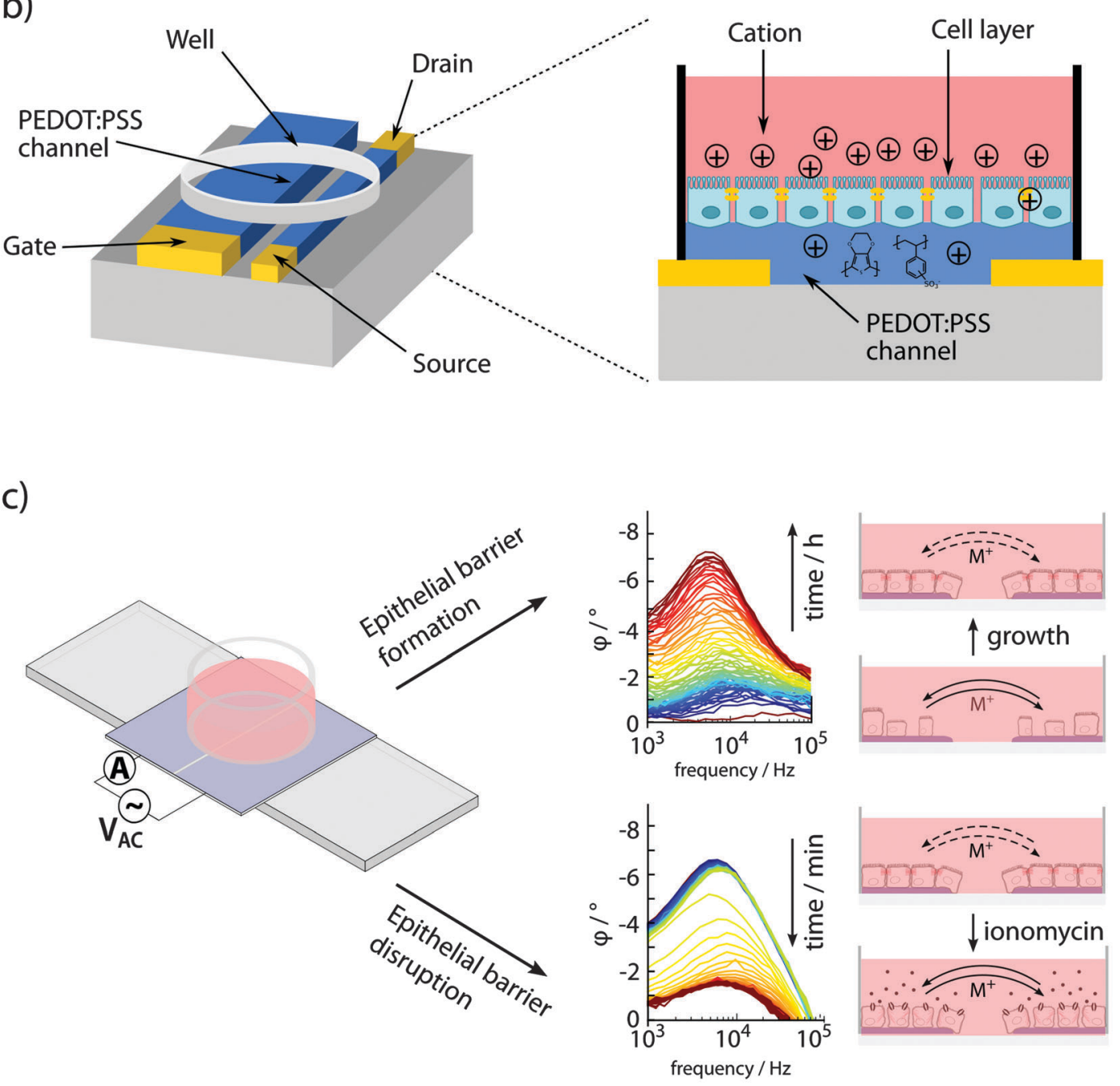

Fig. 5 Sensing of epithelial barrier function. (a) Bacterial pathogens interacting with epithelial cells involve changes in paracellular ion exchange and transepithelial resistance (TER). (b) A planar OECT enabling TER measurement and phase contrast microscopy of cells growing directly on the device. (c) Fully organic planar 2-electrode PAS device to monitor epithelial formation and disruption using PAS and monitoring of $\varphi$ peak amplitude at $10^{3}-10^{5} \mathrm{~Hz}$. Panel (b) is reproduced from Ramuz et al. ${ }^{85}$ and (c) is reproduced and modified from Löffler et al. ${ }^{86}$

are transmitted to recruit immune cells to the site of infection, and the extravasation of polymorphonucleic leukocytes efficiently promotes bacterial clearance within $24 \mathrm{~h}$. Clotting in the peritubular capillaries was identified as an innate defense mechanism, maintaining bacteria at the infection site while avoiding systemic spread and sepsis. ${ }^{81}$ Evidently, the mechanisms 
of bacterial clearance, even at the very localized level of the infection site, is much more complex than any in vitro system could have predicted. To approach this problem, advanced in vitro systems based on organic bioelectronics are being developed.

In in vitro experiments, epithelial barrier integrity can be monitored using an OECT. ${ }^{82-85}$ In a custom-designed well, epithelial cells were grown on the filter of a transwell insert positioned above the OECT channel, with an $\mathrm{Ag} / \mathrm{AgCl}$ gate electrode immersed from the top. The device was continuously addressed with a constant negative drain potential $\left(V_{\mathrm{DS}}=\right.$ $-0.1 \mathrm{~V})$ and pulses of positive gate potential $\left(V_{\mathrm{G}}=0.3 \mathrm{~V}, 1 \mathrm{~s}\right.$ on, $29 \mathrm{~s}$ off), the latter leading to transepithelial resistance (TER)-dependent de-doping of the channel. A tight epithelial layer with high TER limits the de-doping compared to low TER in disrupted epithelium. Since the drain current $I_{\mathrm{D}}$ is proportional to the de-doping of the channel (cations entering to counterbalance the negative charge of PSS, see Fig. 2b), its transient response can be used to monitor the TER. The sensitivity of the device for recording of epithelial integrity ranges from artificial epithelial disruption by addition of high concentrations $\mathrm{H}_{2} \mathrm{O}_{2}$ or EtOH, ${ }^{82}$ and EGTA-mediated $\mathrm{Ca}^{2+}$ chelating, ${ }^{83}$ to recordings of the effect of different strains of Salmonella typhimurium. ${ }^{84}$ A planar re-design of the OECT, this time applying a PEDOT:PSS gate electrode in parallel to the PEDOT:PSS channel, enabled microscopic observation while recording the integrity of the cell layer on the conducting material after addition of EGTA or trypsin (see Fig. 5b). ${ }^{85}$

Visualizing bacterial infections in real-time within the live organ has uncovered the highly dynamic nature of the infection process. Delicate physiological alterations at the local infection site are of immense importance for the outcome of infection. To reveal the molecular details of such processes, automated and quantitative in vitro cell culture technologies are required, allowing continuous recordings of cell cultures over several days while minimizing disturbance of the cells. Recently, phase angle spectroscopy (PAS) based on a 2-electrode PEDOT:PSS device was shown as a novel method for dynamic monitoring of epithelial barrier formation and disruption (see Fig. 5c). ${ }^{86}$ The phase angle $\varphi$ of the current response versus a low sinusoidal $0.1 \mathrm{~V}$ voltage input was determined hourly over a period of 3-4 days, for sinusoidal frequencies between $0.1-10^{5} \mathrm{~Hz}$ in a dish seeded with renal epithelial cells. A peak in $\varphi$ amplitude at $10^{3}-10^{5} \mathrm{~Hz}$ was identified, which corresponded to increased cell capacitance as the epithelial monolayer formed on the polymer electrodes. Transparency of the electrode material facilitated immunofluorescence-based identification of the tight junction protein ZO1, demonstrating that a polarized epithelial monolayer had formed. Addition of an ionophore to the polarized monolayer demonstrated the PAS technology as a valuable tool to also monitor disruption of the barrier function. High-resolution data on the kinetics of membrane-disrupting ionomycin on the polarized epithelium was obtained by quantification of the $\varphi$ peak amplitude, with measurements recorded in the partial spectral range every $30 \mathrm{~s}$. In comparison to simultaneous phase contrast imaging, PAS recordings showed a much higher sensitivity. Besides monitoring cell growth, density and polarity, continuous electronic sensing offers a novel method to optimize timing for cell passage or treatment. Via wireless alerts, the scientist will be immediately informed when cells are ready for passage, or if any other unexpected event occurs, such as early detection of contaminations, slow deterioration and de-differentiation of cells (see Fig. 6). With tissue culture forming the base of personalized regenerative medicine, the combined optical and electronic sensing offered by the fully organic PAS sensor could lead to fully automated systems of high relevance in numerous fields related to cell biology.

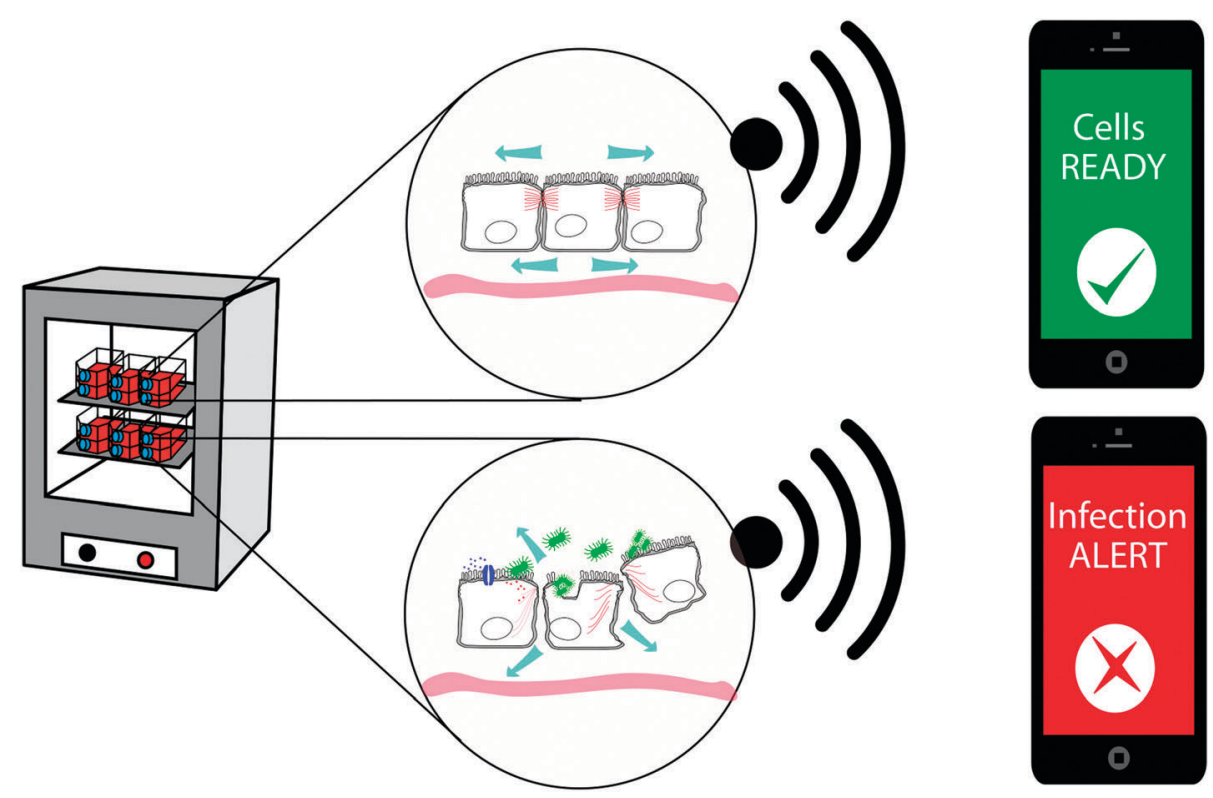

Fig. 6 Potential applications for organic bioelectronic devices as fully automated monitoring and alert systems for cell culture. 


\subsection{Mechanostimulation of transitional epithelium}

Some organs, such as the urinary bladder, are composed of transitional epithelium that accommodates great fluctuations of the volume of the liquid. Contraction and expansion of cells in the transitional epithelium provides the degree of distension needed for the bladder to fluctuate between an empty and a full state. The transitional epithelium also serves as a barrier, with tight junctions protecting the inside of the body from outside exposure. Upon infection, a rise in the interstitial pressure occurs due to increased water uptake into the interstitium. Technologies to enable in vitro studies of mechanotransduction and mechanical stimulation of epithelia are thus highly relevant. In organs and tissues, this has been studied using flexible tissue culture substrates that can be stretched by external means. ${ }^{87}$ However, on the cellular and subcellular level, dedicated technology to apply appropriate mechanical stimuli is limited. Svennersten et al. developed an organic electronic microactuator chip for mechanical stimulation of single cells. ${ }^{30}$ Traditional photolithography and microfabrication was used to generate individually addressable, alternating areas of PPy and SU8 on a silicon wafer. Uroepithelial cells growing on the PPy/SU8 were mechanically stimulated as the polymer expanded due to electrochemical switching. The observed increase of intracellular $\mathrm{Ca}^{2+}$ accounted for the activation of an autocrine ATP signalling pathway associated with mechanical stimulation of cells. The sensitivity of elastic organs to infection under relaxed versus stretched conditions may be easily addressed using this mechanostimulation technology.

\subsection{Treating infection}

Many infections are associated with bacteria adopting a specific lifestyle termed biofilm. This is established when free-living bacteria, so called planktonic bacteria, attach to a biotic or abiotic surface and undergo a transition to the biofilm phenotype. In a biofilm, dense aggregates of bacteria are embedded in large amounts of extracellular matrix and they firmly adhere to a surface. ${ }^{88,89}$ The biofilm structure hides highly immunogenic bacterial cells from the immune system and diffusion of antibiotics into the biofilm is restricted. Further, biofilm formation leads to development of persister phenotypes which slow down metabolism thus negating the mode of action of many antibiotics which require dividing bacteria in order to be effective. ${ }^{90}$ Biofilms are a particular problem on implanted medical devices. Invasive surgeries coupled with the insertion of an implant present both a point of entry for invading bacteria and a surface upon which to attach and initiate biofilm formation. Treating biofilm-associated infections is extremely difficult and high systemic concentrations of antibiotics are administered over a prolonged period of time in order to reach sufficient levels at the site of infection. Even then, biofilms prevent effective killing and, once established, are generally considered as irreversible even under harsh antibiotic treatment and surgical intervention is required. ${ }^{91}$

Major ongoing activities focus on the development of novel surfaces for use in medical devices in order to either reduce the probability of a biofilm formation or to treat a biofilm and allow effective killing of bacteria. The problem is often addressed by enabling antibiotic release directly from a surface. The benefits of such methods are that high concentrations of antibiotic can be delivered to the infection site without requiring invasive surgery or high systemic concentrations of the drugs as is the case with oral or intravenous administration (see Fig. 7a), both of which can compromise the health of the patient. ${ }^{91}$ Antibiotic release from a conducting polymer film raises the possibility of using more cytotoxic antibiotics such as polymyxin $\mathrm{B}$, as the local concentrations can be high while the systemic concentrations are low (see Fig. 7b). ${ }^{92,93}$ The advantage of organic bioelectronic drug release systems is that they feature electronically triggered (on) or controlled (on/off) release of antibiotics, compared to sustained release with common polymer systems. $^{94-100}$ a)

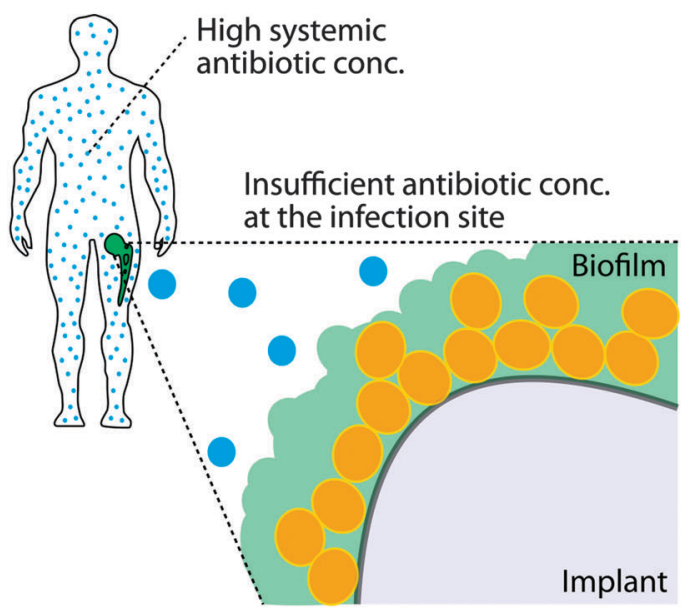

b)

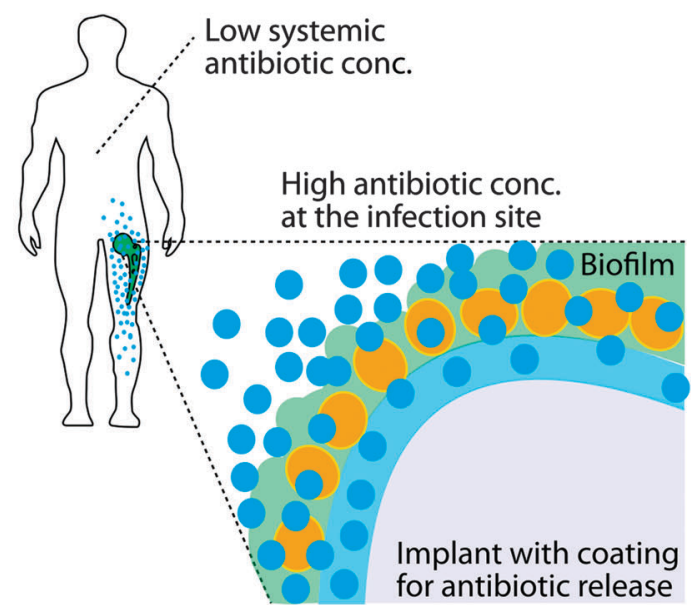

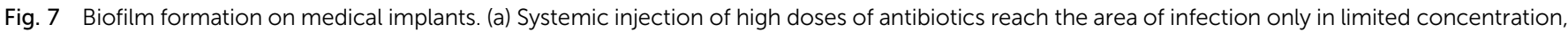

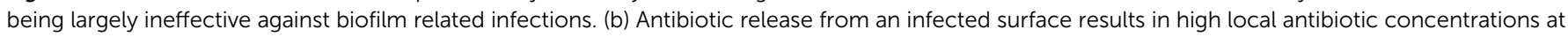
the biofilm, potentially increasing efficiency against biofilm related infections and minimizing systemic effects. 
Electronically triggered drug release from electric-field-responsive PPy nanoparticles was achieved recently by Ge et al. ${ }^{101}$ PPy nanoparticles were prepared by emulsion polymerisation and loaded with fluorescein and daunorubicin. After purification, the polymers were embedded in a temperature responsive hydrogel and injected subcutaneously. An electric field with $-1.5 \mathrm{~V} \mathrm{~cm}^{-1}$ was applied onto the implanted gels via two needle electrodes for $40 \mathrm{~s}$ and, in response, sensitive and dosage-controlled release of drugs was achieved. Electronically controlled drug release from nanoparticles could provide means for repeated, systemic administration to treat chronic infections and inflammation.

Electronically controlled drug release has been demonstrated from PPy films, used as functionalized coatings on implants and prostheses. Functionalized prostheses are developed with drug containing film coatings to prevent infection at the implantation site and improve tissue integration. ${ }^{102} \mathrm{Ti}$ is still the material of choice for bone and joint prostheses in the clinics however, there is a high risk of implant infection due to the invasive surgery required to place the implant. Recurring inflammatory processes at the implant-bone interface prevent ingrowth and lead to implant failure. ${ }^{103}$ Drug release capacities of nanoporous PPy films deposited on $\mathrm{Ti}$ with dexamethasone or penicillin/streptomycin were studied by Sirivisoot et al. They showed that $80 \%$ of the drugs were released on demand during 5 cyclic voltammetry sweeps. ${ }^{104}$ Also, it was shown that, while fibroblast adhesion on the PPy film is inhibited, bone-forming osteoblast adhesion was enhanced on PPy doped with the anti-inflammatory drug dexamethasone. Electrically triggered drug release from antimicrobial- and antiinflammatory-coated devices hindered bacterial and macrophage growth compared with controls. ${ }^{105}$

Despite promising results, loading the polymer by using the drug as a doping agent might be suboptimal, as this method limits the drug loading capacity, and the range of drugs to negatively charged compounds. Nevertheless, it has been shown that electronically triggered release from PPy scaffolds can be achieved with neutral drugs. High surface area PPy scaffolds were shown to significantly enhance the drug loading capacity of PPy delivery devices. ${ }^{106}$ Using these scaffolds, tuneable delivery of the neutral drug progesterone was demonstrated. ${ }^{107}$ It is likely that this drug is physically trapped in the porous polymer and released after switching due to polymer swelling. Based on this assumption, PPy nanowire networks have been prepared electrochemically in which the micro- and nano-gaps separating individual PPy nanowires seem to act as reservoirs for drug storage. ${ }^{108}$ In this system, drug-loading capacity is dependent on the volume of the micro- and nano-vacancies, rather than the doping level. Both hydrophilic and lipophilic drugs can be loaded into the micro- and nano-gaps due to the amphilicity of the PPy nanowire network.

\section{Conclusions}

The global threat of bacterial infection poses a major challenge. This is further complicated by an increasing bacterial resistance to antibiotics. Today, many diverse approaches aiming to reduce and eradicate the morbidity and mortality associated with infection are described. Interdisciplinary efforts have spurred the development of novel tools, able to realistically simulate the high complexity of the in vivo microenvironment. In utilizing the electronic and ionic conductivity of conjugated polymers, organic bioelectronics have been developed that can establish and transiently modulate ionic gradients as well as reversibly tune surface properties and material swelling. These material properties can be used to actively mimic specific microenvironments in human tissues and help decipher the complexity of multi-layered biological responses, which are observed states of infection in the human host. The organic bioelectronics research field is advancing at a high pace with further development of material properties, design of devices, and expansion of the molecular transport repertoire. This collective effort will aid in making organic bioelectronics devices useful for a number of applications in vitro. Also, one can foresee a number of therapeutic areas that would benefit from the technology, as it becomes established as a candidate for the next generation of implantable biomedical devices.

\section{Acknowledgements}

We thank all our partners for fruitful collaborations building a highly interdisciplinary research environment. Research in the ARD laboratory is supported by the Swedish Medical Nanoscience Center (www.medicalnanoscience.se), Carl Bennet AB, VINNOVA, Karolinska Institutet, and Familjen Erling-Perssons Stiftelse.

\section{References}

1 M. Kim, H. Ashida, M. Ogawa, Y. Yoshikawa, H. Mimuro and C. Sasakawa, Cell Host Microbe, 2010, 8, 20-35.

2 P.-O. Méthot and S. Alizon, Virulence, 2014, 5, 775-785.

3 P. Lüthje and A. Brauner, Adv. Microb. Physiol., 2014, 65, 337-372.

4 K. Melican and A. Richter-Dahlfors, Curr. Opin. Microbiol., 2009, 12, 31-36.

5 A. Richter-Dahlfors, M. Rhen and K. Udekwu, Curr. Opin. Microbiol., 2012, 15, 15-22.

6 F. X. Choong, J. Regberg, K. I. Udekwu and A. RichterDahlfors, Future Microbiol., 2012, 7, 519-533.

7 M. Berggren and A. Richter-Dahlfors, Adv. Mater., 2007, 19, 3201-3213.

8 A. Richter-Dahlfors and P. Kjäll, Biochim. Biophys. Acta, 2011, 1810, 237-238.

9 R. Owens, P. Kjall, A. Richter-Dahlfors and F. Cicoira, Biochim. Biophys. Acta, 2013, 1830, 4283-4285.

10 K. C. Larsson, P. Kjäll and A. Richter-Dahlfors, Biochim. Biophys. Acta, 2013, 1830, 4334-4344.

11 M. Muskovich and C. J. Bettinger, Adv. Healthcare Mater., 2012, 1, 248-266.

12 J. L. Brédas, A. J. Heeger and F. Wudl, J. Chem. Phys., 1986, 85, 4673.

13 A. J. Heeger, Chem. Soc. Rev., 2010, 39, 2354-2371. 
14 I. McCulloch, M. Heeney, M. L. Chabinyc, D. DeLongchamp, R. J. Kline, M. Cölle, W. Duffy, D. Fischer, D. Gundlach, B. Hamadani, R. Hamilton, L. Richter, A. Salleo, M. Shkunov, D. Sparrowe, S. Tierney and W. Zhang, Adv. Mater., 2009, 21, 1091-1109.

15 A. G. MacDiarmid, R. J. Mammone, R. B. Kaner, S. J. Porter, R. Pethig, A. J. Heeger and D. R. Rosseinsky, Philos. Trans. R. Soc., A, 1985, 314, 3-15.

16 H.-S. Park, S.-J. Ko, J.-S. Park, J. Y. Kim and H.-K. Song, Sci. Rep., 2013, 3, 2454.

17 R. Kroon, M. Lenes, J. C. Hummelen, P. W. M. Blom and B. de Boer, Polym. Rev., 2008, 48, 531-582.

18 J. Roncali, Macromol. Rapid Commun., 2007, 28, 1761-1775.

19 C. L. Chochos and S. A. Choulis, Prog. Polym. Sci., 2011, 36, 1326-1414.

20 L. Dai, Intelligent Macromolecules for Smart Devices: From Materials Synthesis to Device Applications, Springer Science \& Business Media, 2004.

21 X. Wang, M. Berggren and O. Inganäs, Langmuir, 2008, 24, 5942-5948.

22 K. Svennersten, K. C. Larsson, M. Berggren and A. RichterDahlfors, Biochim. Biophys. Acta, 2011, 1810, 276-285.

23 M. H. Bolin, K. Svennersten, X. Wang, I. S. Chronakis, A. Richter-Dahlfors, E. W. H. Jager and M. Berggren, Sens. Actuators, B, 2009, 142, 451-456.

24 M. Bolin, K. Svennersten, D. Nilsson, A. Sawatdee, E. W. H. Jager, A. Richter-Dahlfors and M. Berggren, Adv. Mater., 2009, 21, 4379-4382.

25 K. Svennersten, M. H. Bolin, E. W. H. Jager, M. Berggren and A. Richter-Dahlfors, Biomaterials, 2009, 30, 6257-6264.

26 K. Naoi, J. Electrochem. Soc., 1991, 138, 440.

27 V. Pillay, T.-S. Tsai, Y. E. Choonara, L. C. du Toit, P. Kumar, G. Modi, D. Naidoo, L. K. Tomar, C. Tyagi and V. M. K. Ndesendo, J. Biomed. Mater. Res., Part A, 2014, 102, 2039-2054.

28 M. R. Gandhi, P. Murray, G. M. Spinks and G. G. Wallace, Synth. Met., 1995, 73, 247-256.

29 T. F. Otero and J. G. Martinez, Adv. Funct. Mater., 2014, 24, 1259-1264.

30 K. Svennersten, M. Berggren, A. Richter-Dahlfors and E. W. H. Jager, Lab Chip, 2011, 11, 3287-3293.

31 D. Summerlot, A. Kumar, S. Das, L. Goldstein, S. Seal, D. Diaz and H. J. Cho, Procedia Eng., 2011, 25, 1457-1460.

32 M. Gerard, A. Chaubey and B. D. Malhotra, Biosens. Bioelectron., 2002, 17, 345-359.

33 S. Gomez-Carretero and P. Kjäll, Medical Applications of Organic Bioelectronics, Organic Electronics, Wiley-VCH Verlag GmbH \& Co. KGaA, Weinheim, Germany, 2013, ch. 3.

34 J. Boekel, O. Källskog, M. Rydén-Aulin, M. Rhen and A. Richter-Dahlfors, BMC Genomics, 2011, 12, 123.

35 M. H. V Van Regenmortel, EMBO Rep., 2004, 5, 1016-1020.

36 H. Kaji, G. Camci-Unal, R. Langer and A. Khademhosseini, Biochim. Biophys. Acta, 2011, 1810, 239-250.

37 A.-S. Andersson, F. Bäckhed, A. von Euler, A. RichterDahlfors, D. Sutherland and B. Kasemo, Biomaterials, 2003, 24, 3427-3436.
38 E. K. F. Yim, R. M. Reano, S. W. Pang, A. F. Yee, C. S. Chen and K. W. Leong, Biomaterials, 2005, 26, 5405-5413.

39 X. Wang, K. Ye, Z. Li, C. Yan and J. Ding, Organogenesis, 2013, 9, 280-286.

40 P. J. Molino, M. J. Higgins, P. C. Innis, R. M. I. Kapsa and G. G. Wallace, Langmuir, 2012, 28, 8433-8445.

41 F. Greco, T. Fujie, L. Ricotti, S. Taccola, B. Mazzolai and V. Mattoli, ACS Appl. Mater. Interfaces, 2013, 5, 573-584.

42 H. Klauk, Chem. Soc. Rev., 2010, 39, 2643-2666.

43 L. Kergoat, B. Piro, M. Berggren, G. Horowitz and M.-C. Pham, Anal. Bioanal. Chem., 2012, 402, 1813-1826.

44 P. Lin, F. Yan and H. L. W. Chan, ACS Appl. Mater. Interfaces, 2010, 2, 1637-1641.

45 D. Nilsson, Sens. Actuators, B, 2002, 86, 193-197.

46 S. T. Plummer, Q. Wang, P. W. Bohn, R. Stockton and M. A. Schwartz, Langmuir, 2003, 19, 7528-7536.

47 K. Melican, J. Boekel, M. Ryden-Aulin and A. RichterDahlfors, Crit. Rev. Immunol., 2010, 30, 107-117.

48 W. S. Yeo, C. D. Hodneland and M. Mrksich, ChemBioChem, 2001, 2, 590-593.

49 M. N. Yousaf, B. T. Houseman and M. Mrksich, Angew. Chem., Int. Ed., 2001, 40, 1093-1096.

50 R. Inaba, A. Khademhosseini, H. Suzuki and J. Fukuda, Biomaterials, 2009, 30, 3573-3579.

51 Y. Seto, R. Inaba, T. Okuyama, F. Sassa, H. Suzuki and J. Fukuda, Biomaterials, 2010, 31, 2209-2215.

52 Z. Tang, Y. Wang, P. Podsiadlo and N. A. Kotov, Adv. Mater., 2006, 18, 3203-3224.

53 O. Guillaume-Gentil, Y. Akiyama, M. Schuler, C. Tang, M. Textor, M. Yamato, T. Okano and J. Vörös, Adv. Mater., 2008, 20, 560-565.

54 O. Guillaume-Gentil, M. Gabi, M. Zenobi-Wong and J. Vörös, Biomed. Microdevices, 2011, 13, 221-230.

55 K. M. Persson, R. Karlsson, K. Svennersten, S. Löffler, E. W. H. Jager, A. Richter-Dahlfors, P. Konradsson and M. Berggren, Adv. Mater., 2011, 23, 4403-4408.

56 E. M. Thaning, M. L. M. Asplund, T. A. Nyberg, O. W. Inganäs and H. von Holst, J. Biomed. Mater. Res., Part B, 2010, 93, 407-415.

57 Y. Niv and G. M. Fraser, J. Clin. Gastroenterol., 2002, 35, 5-8.

58 M. Johansson, I. Synnerstad and L. Holm, Gastroenterology, 2000, 119, 1297-1304.

59 C. Schade, G. Flemström and L. Holm, Gastroenterology, 1994, 107, 180-188.

60 M. A. Croxen, G. Sisson, R. Melano and P. S. Hoffman, J. Bacteriol., 2006, 188, 2656-2665.

61 E. Goers Sweeney, J. N. Henderson, J. Goers, C. Wreden, K. G. Hicks, J. K. Foster, R. Parthasarathy, S. J. Remington and K. Guillemin, Structure, 2012, 20, 1177-1188.

62 L. E. Wroblewski, R. M. Peek and K. T. Wilson, Clin. Microbiol. Rev., 2010, 23, 713-739.

63 J. Isaksson, P. Kjäll, D. Nilsson, N. D. Robinson, M. Berggren and A. Richter-Dahlfors, Nat. Mater., 2007, 6, 673-679.

64 J. Isaksson, D. Nilsson, P. Kjäll, N. D. Robinson, A. RichterDahlfors and M. Berggren, Org. Electron., 2008, 9, 303-309. 
65 D. T. Simon, S. Kurup, K. C. Larsson, R. Hori, K. Tybrandt, M. Goiny, E. W. H. Jager, M. Berggren, B. Canlon and A. Richter-Dahlfors, Nat. Mater., 2009, 8, 742-746.

66 J. Isaksson, D. Nilsson, P. Kjäll, N. D. Robinson, A. RichterDahlfors and M. Berggren, Org. Electron., 2008, 9, 303-309.

67 P. Uhlén, A. Laestadius, T. Jahnukainen, T. Söderblom, F. Bäckhed, G. Celsi, H. Brismar, S. Normark, A. Aperia and A. Richter-Dahlfors, Nature, 2000, 405, 694-697.

68 C. Oxhamre, A. Richter-Dahlfors, V. P. Zhdanov and B. Kasemo, Biophys. J., 2005, 88, 2976-2981.

69 L. E. Månsson, K. Melican, J. Boekel, R. M. Sandoval, I. Hautefort, G. A. Tanner, B. A. Molitoris and A. Richterdahlfors, Cell. Microbiol., 2007, 9, 413-424.

70 M. J. Berridge, M. D. Bootman and H. L. Roderick, Nat. Rev. Mol. Cell Biol., 2003, 4, 517-529.

71 T. Söderblom, A. Laestadius, C. Oxhamre, A. Aperia and A. Richter-Dahlfors, Int. J. Med. Microbiol., 2002, 291, 511-515.

72 T. Söderblom, C. Oxhamre, S. N. Wai, P. Uhlén, A. Aperia, B. E. Uhlin and A. Richter-Dahlfors, Cell. Microbiol., 2005, 7, 779-788.

73 P. Uhlén, A. Laestadius, T. Jahnukainen, T. Söderblom, F. Bäckhed, G. Celsi, H. Brismar, S. Normark, A. Aperia and A. Richter-Dahlfors, Nature, 2000, 405, 694-697.

74 K. Tybrandt, K. C. Larsson, S. Kurup, D. T. Simon, P. Kjäll, J. Isaksson, M. Sandberg, E. W. H. Jager, A. Richter-Dahlfors and M. Berggren, Adv. Mater., 2009, 21, 4442-4446.

75 M. B. Pepys and G. M. Hirschfield, J. Clin. Invest., 2003, 111, 1805-1812.

76 T. Goda, P. Kjall, K. Ishihara, A. Richter-Dahlfors and Y. Miyahara, Adv. Healthcare Mater., 2014, 3, 1733-1738.

77 L. W. Peterson and D. Artis, Nat. Rev. Immunol., 2014, 14, 141-153.

78 A. M. Marchiando, W. V. Graham and J. R. Turner, Annu. Rev. Pathol.: Mech. Dis., 2010, 5, 119-144.

79 J. A. Guttman and B. B. Finlay, Biochim. Biophys. Acta, 2009, 1788, 832-841.

80 K. Melican, J. Boekel, L. E. Månsson, R. M. Sandoval, G. A. Tanner, O. Källskog, F. Palm, B. A. Molitoris and A. Richter-Dahlfors, Cell. Microbiol., 2008, 10, 1987-1998.

81 K. Melican, J. Boekel, L. E. Månsson, R. M. Sandoval, G. A. Tanner, O. Källskog, F. Palm, B. A. Molitoris and A. Richter-Dahlfors, Cell. Microbiol., 2008, 10, 1987-1998.

82 L. H. Jimison, S. A. Tria, D. Khodagholy, M. Gurfinkel, E. Lanzarini, A. Hama, G. G. Malliaras and R. M. Owens, Adv. Mater., 2012, 24, 5919-5923.

83 S. Tria, L. H. Jimison, A. Hama, M. Bongo and R. M. Owens, Biosensors, 2013, 3, 44-57.

84 S. A. Tria, M. Ramuz, M. Huerta, P. Leleux, J. Rivnay, L. H. Jimison, A. Hama, G. G. Malliaras and R. M. Owens, Adv. Healthcare Mater., 2014, 3, 1053-1060.
85 M. Ramuz, A. Hama, M. Huerta, J. Rivnay, P. Leleux and R. M. Owens, Adv. Mater., 2014, 26, 7083-7090.

86 S. Löffler and A. Richter-Dahlfors, J. Mater. Chem. B, 2015, DOI: 10.1039/C5TB00381D.

87 F. J. Alenghat, S. M. Nauli, R. Kolb, J. Zhou and D. E. Ingber, Exp. Cell Res., 2004, 301, 23-30.

88 N. Høiby, T. Bjarnsholt, M. Givskov, S. Molin and O. Ciofu, Int. J. Antimicrob. Agents, 2010, 35, 322-332.

89 T. Bjarnsholt, M. Alhede, M. Alhede, S. R. EickhardtSørensen, C. Moser, M. Kühl, P. Ø. Jensen and N. Høiby, Trends Microbiol., 2013, 21, 466-474.

90 I. Keren, D. Shah, A. Spoering, N. Kaldalu and K. Lewis, J. Bacteriol., 2004, 186, 8172-8180.

91 R. O. Darouiche, N. Engl. J. Med., 2004, 350, 1422-1429.

92 M. Evans, Ann. Pharmacother., 1999, 33, 960-967.

93 D. Svirskis, J. Travas-Sejdic, A. Rodgers and S. Garg, J. Controlled Release, 2010, 146, 6-15.

94 U. Brohede, J. Forsgren, S. Roos, A. Mihranyan, H. Engqvist and M. Strømme, J. Mater. Sci.: Mater. Med., 2009, 20, 1859-1867.

95 N. Blanchemain, S. Haulon, F. Boschin, E. MarconBachari, M. Traisnel, M. Morcellet, H. F. Hildebrand and B. Martel, Biomol. Eng., 2007, 24, 149-153.

96 G. Buschle-Diller, J. Cooper, Z. Xie, Y. Wu, J. Waldrup and X. Ren, Cellulose, 2007, 14, 553-562.

97 N. Dunne, J. Hill, P. McAfee, K. Todd, R. Kirkpatrick, M. Tunney and S. Patrick, Acta Orthop., 2007, 78, 774-785.

98 K. Kim, Y. K. Luu, C. Chang, D. Fang, B. S. Hsiao, B. Chu and M. Hadjiargyrou, J. Controlled Release, 2004, 98, 47-56.

99 H. F. Chuang, R. C. Smith and P. T. Hammond, Biomacromolecules, 2008, 9, 1660-1668.

100 M. V Risbud, A. A. Hardikar, S. V Bhat and R. R. Bhonde, J. Controlled Release, 2000, 68, 23-30.

101 J. Ge, E. Neofytou, T. J. Cahill, R. E. Beygui and R. N. Zare, ACS Nano, 2012, 6, 227-233.

102 A. N. Zelikin, ACS Nano, 2010, 4, 2494-2509.

103 D. M. Brunette, P. Tengvall, M. Textor and P. Thomsen, Titanium in Medicine: Material Science, Surface Science, Engineering, Biological Responses, and Medical Applications, Springer Science \& Business Media, 2001.

104 S. Sirivisoot, R. Pareta and T. J. Webster, Nanotechnology, 2011, 22, 085101.

105 S. Sirivisoot, R. A. Pareta and T. J. Webster, J. Biomed. Mater. Res., Part A, 2011, 99, 586-597.

106 M. Sharma, G. I. N. Waterhouse, S. W. C. Loader, S. Garg and D. Svirskis, Int. J. Pharm., 2013, 443, 163-168.

107 D. Svirskis, M. Sharma, Y. Yu and S. Garg, Ther. Delivery, 2013, 4, 307-313.

108 S. Jiang, Y. Sun, X. Cui, X. Huang, Y. He, S. Ji, W. Shi and D. Ge, Synth. Met., 2013, 163, 19-23. 